\title{
PLACENTAL TRANSFUSION STRATEGIES IN PRETERM INFANTS < 1000 G BW: META- ANALYSIS OF SHORT AND LONG TERM OUTCOMES
}

\author{
D. Batra ${ }^{1}$, S. Ghavam ${ }^{2}$, H. Kirpalani ${ }^{2}$, J. Mercer ${ }^{3}$, K. Amir ${ }^{4}$, S. Hosono ${ }^{5}$, H. Rabe ${ }^{6}$ \\ ${ }^{I}$ Neonatology, Croydon University Hospital NHS Trust, Croydon, UK, ${ }^{2}$ Neonatology, Children's Hospital of \\ Philadelphia, Philadelphia, PA, ${ }^{3}$ College of Nursing, University of Rhode Island, Kingston, RI, USA, \\ ${ }^{4}$ Neonatology, Bnai-Zion Medical Center, Haifa, Israel, ${ }^{5}$ Paediatrics and Child Health, Nihon University \\ School of Medicine, Tokyo, Japan, ${ }^{6}$ Neonatology, Brighton and Sussex University Hospitals NHS Trust, \\ Brighton, UK
}

Background: Delayed umbilical cord clamping (DCC) or umbilical cord milking (UCM) in ELBW neonates reduces the number of blood transfusions. DCC is now a common practice in term infants, but the outcomes of preterm infants below $1000 \mathrm{~g}$ subjected to DCC or UCM are unclear.

Objective: A meta-analysis of short and long term outcomes of infants $<30$ weeks GA and $<1000 \mathrm{~g}$ BW randomised to either DCC or UCM as compared to immediate cord clamping (ICC).

Methods: Twi independent investigators searched the literature for trials that randomised preterm infants $<$ $1000 \mathrm{~g}$ BW to either experimental(DCC or UCM) or control(ICC) groups. Primary outcome was 24 month Neurodevelopmental Impairment(NDI) using standardised outcomes; secondary outcomes included transfusions. Additional information was requested of authors. Data was summarised by RevMan5 as weighted mean difference(WMD) and 95\%CI.

Results: Searches yielded 15 studies, 6 were included, describing 108 infants. Data on NDI was limited and could not be pooled. One study (Mercer) recorded Bayley at 7 months for 27 infants(WMD MDI -4.40; CI $18.02,+9.22 ; \mathrm{p}=\mathrm{NS}$ ). Another study (Hosono) found no significant differences in disability rates(UCM 19\% vs ICC $27 \%$; $\mathrm{p}=\mathrm{NS}$ ) at $24 \mathrm{months}$ using a Japanese scale.

Short term benefits of DCC/UCM included better mean BP on admission(WMD -4.9;CI -5.58,-4.22) and hemoglobin(WMD 3.71;CI 3.94, 3.47). Other benefits included reduced number of blood transfusions and days on ventilator.

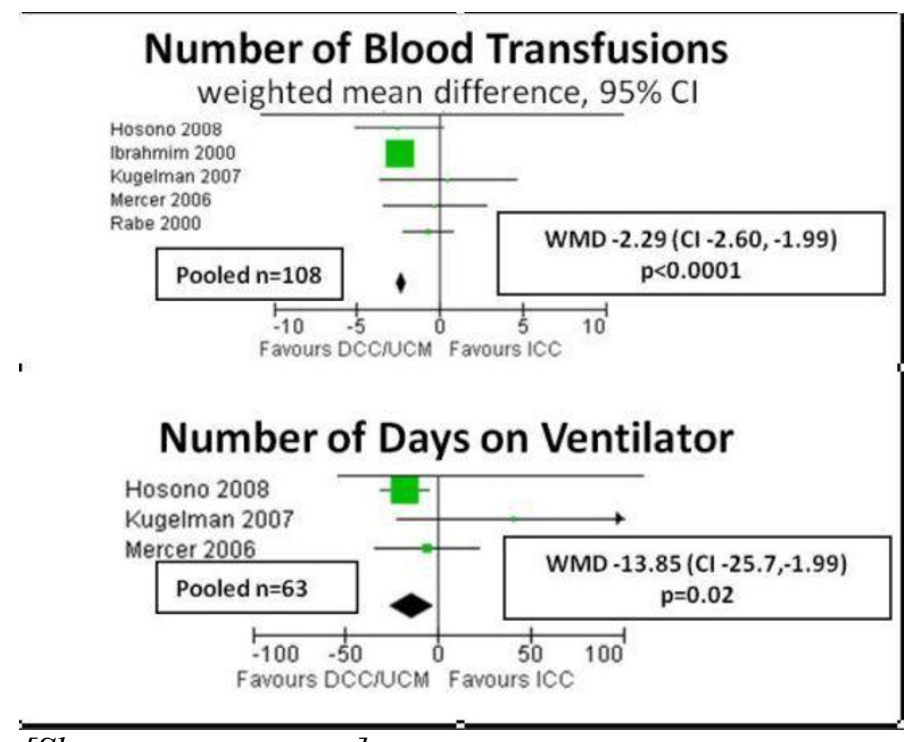

[Short-term outcomes]

Conclusions: Only one study reports 24 month NDI, and no pooling is possible. Short term benefits of DCC include rise in hemoglobin, decreased number of transfusions and shorter days on ventilator. 